\title{
Analysis on the Magnetic Shielding Effectiveness of DC Current Comparator
}

\author{
Jiayun Song, Pan Pan, Qizheng Ji, Shuqiang Wang, Biye Liu \\ Beijing Orient Institute of Measurement \& Test, Beijing, China \\ Email: songjiayun@sohu.com
}

Received 8 December 2015; accepted 26 February 2016; published 29 February 2016

\begin{abstract}
There are magnetic interference problems in the applications of DC current comparator. Analysis on the magnetic effectiveness which is applied by the external magnetic field is introduced in this paper. The effectiveness is proved by the actual results which are compared with the magneticcircuit method and the finite element method. In addition, the reference comment is given which can be used in the practical work of DC current comparator shield design.
\end{abstract}

\section{Keywords}

Magnetic Shielding Effectiveness, DC Current Comparator, the Magnetic-Circuit Method, the Finite Element Method

\section{Introduction}

Magnetic shielding is one of the technical difficulties in the design of the DC current comparator (DCC), since there is always a magnetic field in the environmental conditions where the DCC is used, so it's very necessary to find a way to estimate the shielding effectiveness of the external magnetic field.

The total magnetic flux in the DCC is divided into two parts which is consist of the working flux and the interference flux, and the voltage produced by the detecting coil is the result of the two parts together. When the current to be measured is zero, the working magnetic flux is zero, however, the interference flux maybe remain at the same time. This may result in error even wrong that the output of the detecting coil is not zero [1]-[3]. The use of the magnetic shielding can reduce the magnetic error effectively since the interference flux is very difficult to reach the detecting coil over this.

It always takes the method that depends on experience in the design of magnetic shielding used in the DCC. Although there are many different methods to estimate the shielding effectiveness, the actual work is not consistent with the calculation and simulation results for many different reasons, for example, the shield shape, the band distribution of the disturbing magnetic field, and so on [4] [5]. On the other hand, the reliability of the simulation results is not so good because a lot of approximation is adopted in the calculation and simulation.

Therefore, the practical significance is considerable if we can provide a way by which the magnetic shielding effectiveness of the DCC can be calculated effectively. The shielding effectiveness is contrasted and analyzed between the magnetic-circuit method and the finite element method in this paper, and the advice which can be 
used in the practical work is supplied.

The magnetic shielding effectiveness [6] is commonly defined as the ratio of the magnetic field intensity at a point without the shield to the magnetic field intensity with the shield at the same position. It's given as follows.

$$
S=\frac{H_{0}}{H_{\mathrm{T}}}
$$

where $s$ is the magnetic shielding effectiveness, $H_{0}$ is the magnetic field intensity without shield, $H_{\mathrm{T}}$ is the magnetic field intensity with shield.

\section{The Magnetic Shielding Effectiveness Analysis in Applied Magnetic Field}

\subsection{Applied Axial Magnetic Field}

The shielding effectiveness in the applied axial magnetic field is shown in Figure 1.

The permeability of magnetic shielding materials is very high, so most of magnetic field line goes through the shielding layer, the magnetic field line which can reach the detecting coil is rare. The permeability of magnetic shielding materials is higher, or the shielding layer is thicker, the shielding effectiveness is better.

The model of magnetic shield in the applied axial magnetic field is shown in Figure 2. where $a$ is the thickness, $c$ is the cross section length.

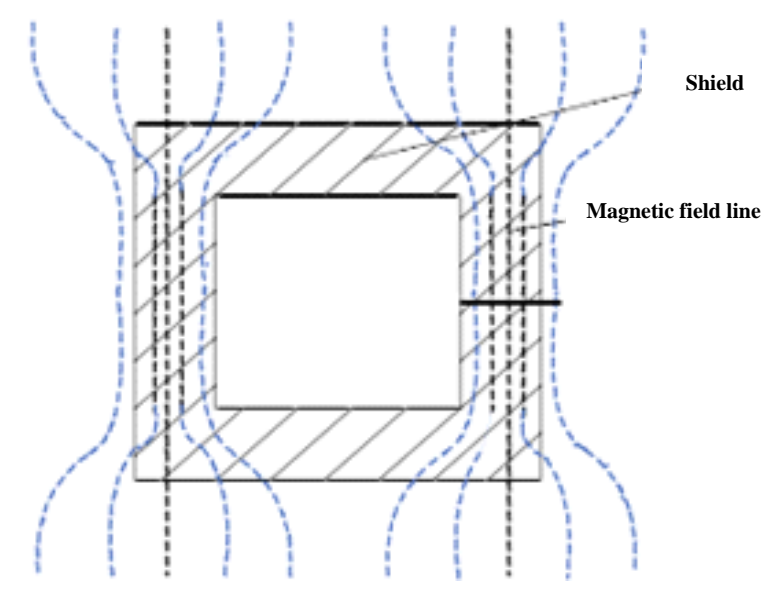

Figure 1. The distribution of magnetic field line in the axial magnetic field.

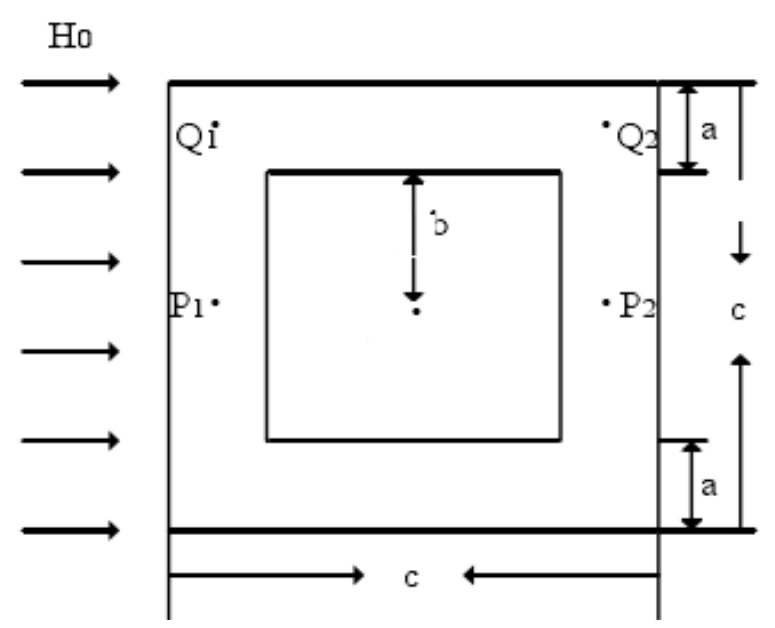

Figure 2. The model of magnetic shield in the axial magnetic field. 
The formula of shielding effectiveness which is derived by the magnetic-circuit method is shown as follows.

$$
S_{1}=\frac{H_{0}}{H_{\mathrm{T}}}=\frac{8}{3} \cdot \frac{\mu_{\mathrm{s}}}{\mu_{0}} \cdot \frac{a b}{c(c-a)}-\frac{2 a}{c}+1=\frac{4}{3} \cdot \mu_{\mathrm{r}} \cdot \frac{a(c-2 a)}{c(c-a)}-\frac{2 a}{c}+1
$$

where $S_{1}$ is the shielding effectiveness, $H_{0}$ is magnetic field intensity outside of the shield, $H_{\mathrm{T}}$ is magnetic field intensity inside of the shield, $\mu_{\mathrm{s}}$ is the shield permeability, $\mu_{0}$ is the air-gap permeability inside of the shield, $\mu_{\mathrm{r}}$ is the relative permeability of the shield.

In the following, the shielding effectiveness can be calculated by the finite element method. The three-dimensional model can be simplified to two-dimensional model in the applied axial magnetic field since the magnetic shield analyzed is the axisymmetric structure. The two-dimensional calculation model is shown in Figure 3.

The magnetic field line is shown in Figure 4 when the shield is placed in the applied axial magnetic field.

As shown above, the magnetic field line is almost concentrated in the shield. This result fits the facts. And then, the shielding effectiveness in different conditions will be demonstrated.

1) Different shielding materials

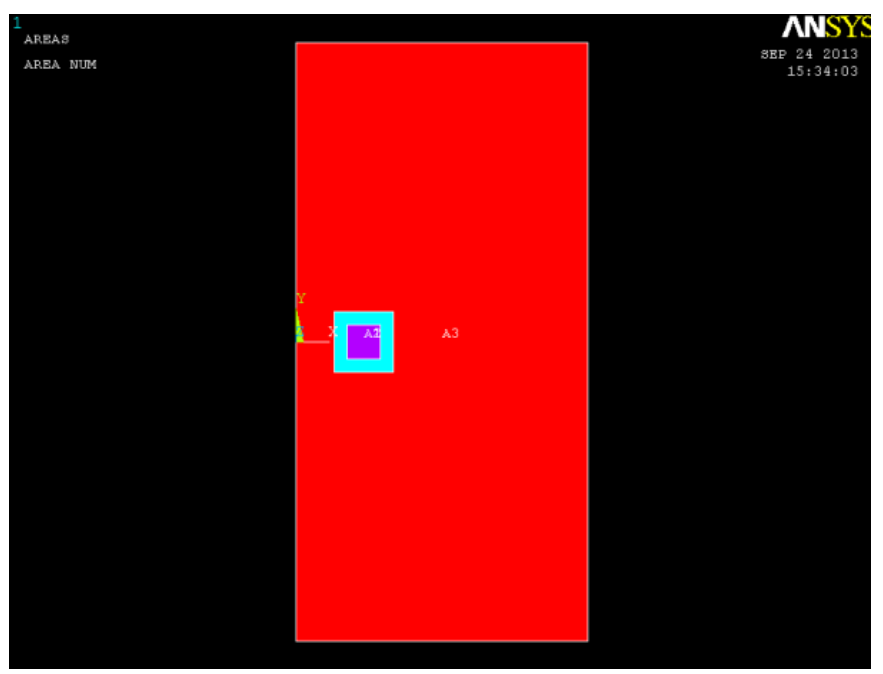

Figure 3. The two-dimensional calculation model with the method of finite element method.

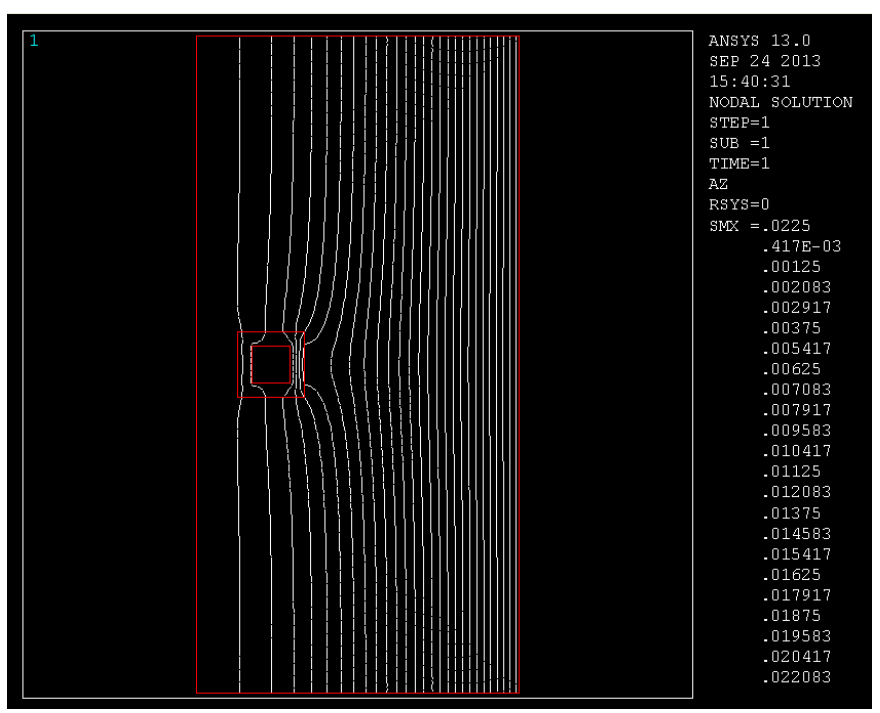

Figure 4. The magnetic field line in the applied axial magnetic field. 
Here, shielding effectiveness with different materials is compared, the results on which the magnetic-circuit method and the finite element method are used respectively are shown in Figure 5.

As shown above, the shielding effectiveness by the finite element method improves with the increase of permeability when the permeability of the shielding material is less than 1000, this result is consistent with the curve by the magnetic-circuit method. While, the shielding effectiveness increases slowly when the permeability continues to increase, and the shielding effectiveness begins to decline when the permeability is more than 2000. This is matched with the actual situation because the shield is saturated when the permeability increases to a certain value.

2) Different thickness of shield

The shielding effectiveness with different thickness of shield which is compared with the magnetic-circuit and the finite element method is shown in Figure 6.

As shown above, the shielding effectiveness firstly increases and then decreases with the increase of thickness.

3) Different cross section length of shield

The shielding effectiveness with different cross section length of shield which is compared with the magnetic-circuit and the finite element method is shown in Figure 7.

As shown above, the shielding effectiveness will reduce with the increase of the cross section length within the effective range.

\subsection{Applied Radial Magnetic Field}

The shielding effectiveness in the applied radial magnetic field is shown in Figure 8.

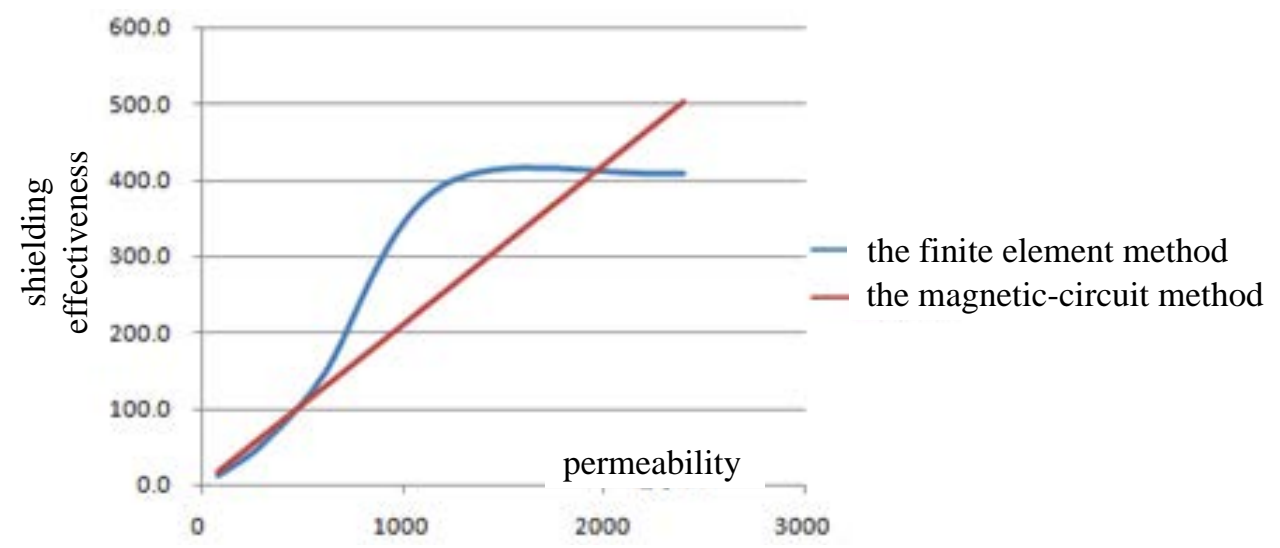

Figure 5. The relationship between the shielding effectiveness and permeability in the applied axial magnetic field.

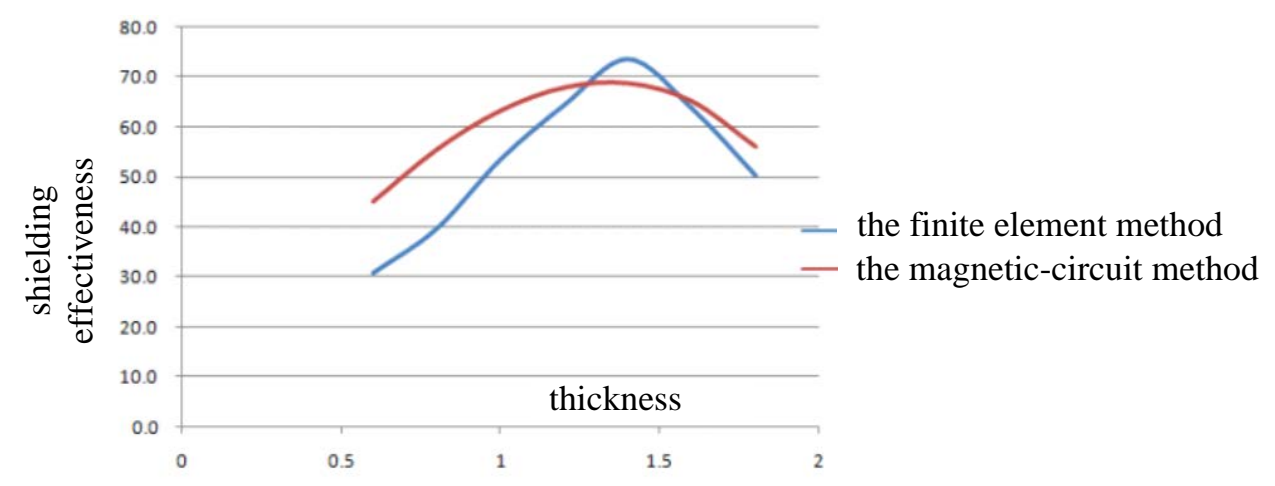

Figure 6. The relationship between the shielding effectiveness and the thickness of shield in the applied axial magnetic field. 


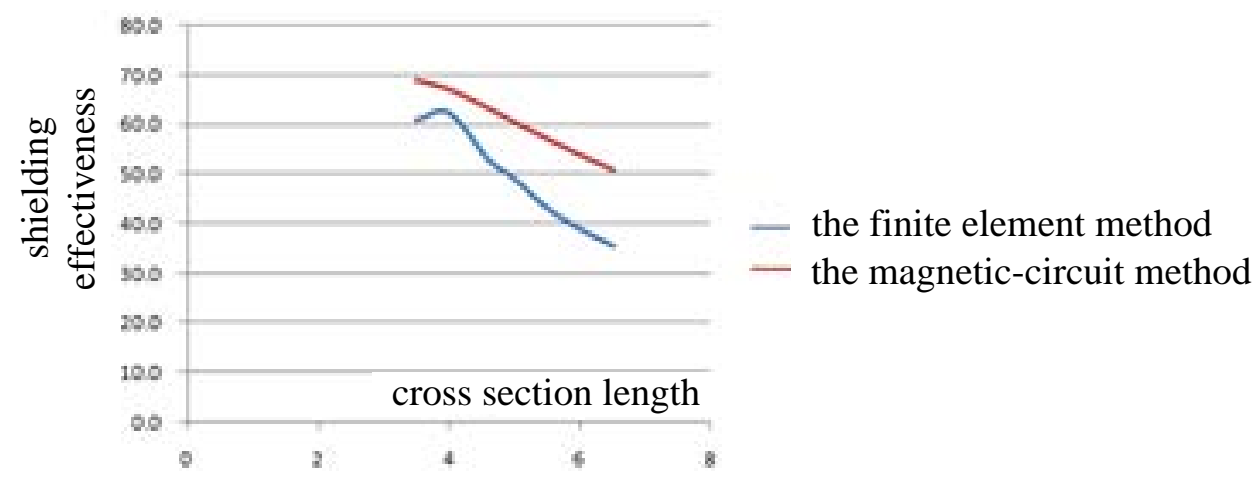

Figure 7. The relationship between the shielding effectiveness and the cross section length of shield in the applied axial magnetic field.

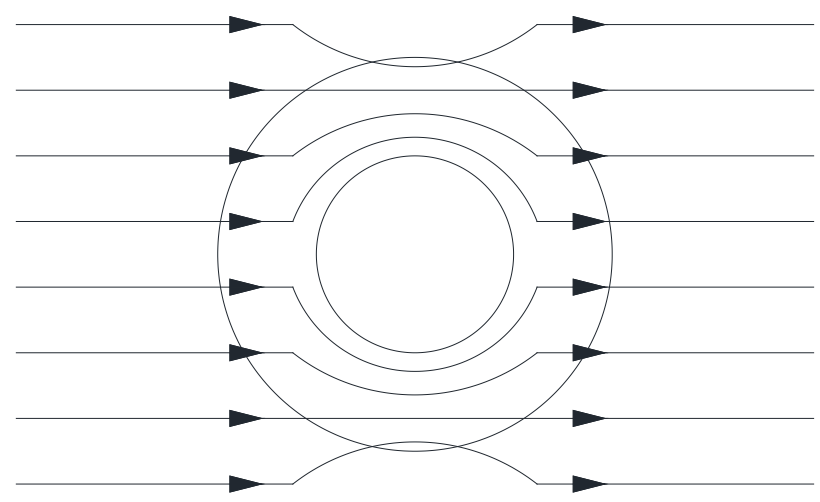

Figure 8. The distribution of magnetic field line in the radial magnetic field.

The model of magnetic shield in the applied radial magnetic field is shown in Figure 9, where $a$ is the thickness, $c$ is the cross section length, $b$ is half of the air gap length, $l$ is the inner semicircle length of the shield.

The formula of shielding effectiveness which is derived by the magnetic-circuit method is shown as follows.

$$
S_{2}=\frac{\Phi_{0}}{\Phi_{\mathrm{T}}}=\frac{\Phi_{\mathrm{T}}+\Phi_{\mathrm{S}}}{\Phi_{\mathrm{T}}}=1+\frac{\Phi_{\mathrm{S}}}{\Phi_{\mathrm{T}}}=\mu_{\mathrm{r}} \times \frac{24 a(c-a)}{\left(2 r_{1}+c\right)^{2} \pi^{2}}+1
$$

where $\Phi_{0}$ is the flux flowing to shield, $\Phi_{\mathrm{S}}$ is the flux flowing through shield, $\Phi_{\mathrm{T}}$ is the leakage flux.

In the following, the shielding effectiveness will be calculated by the finite element method. The three-dimensional model can't be simplified to two-dimensional model in radial magnetic field since the magnetic shield analyzed is not the axisymmetric structure by this time. The three-dimensional calculation model is shown in Figure 10.

The subdivision of the shield in three-dimensional view by the finite element method is shown in Figure 11.

And then we put the shield into the external radial magnetic field, now the distribution of the magnetic field in the shield is shown in Figure 12.

As shown above, the distribution of the magnetic field is changed when the shield is placed. Also, the shielding effectiveness in different conditions will be demonstrated.

1) Different shielding materials

The results on which the magnetic-circuit method and the finite element method are used respectively in the radial magnetic are shown in Figure 13.

As shown above, the shielding effectiveness improves with the increase of permeability in the applied radial magnetic field, the results are consistent with the magnetic-circuit method and the finite element method.

2) Different thickness of shield 


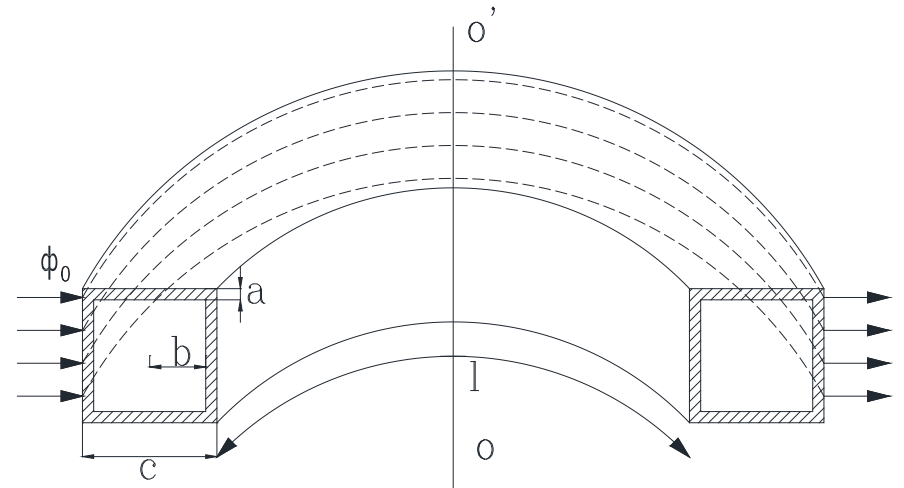

Figure 9. The model of magnetic shield in the radial magnetic field.

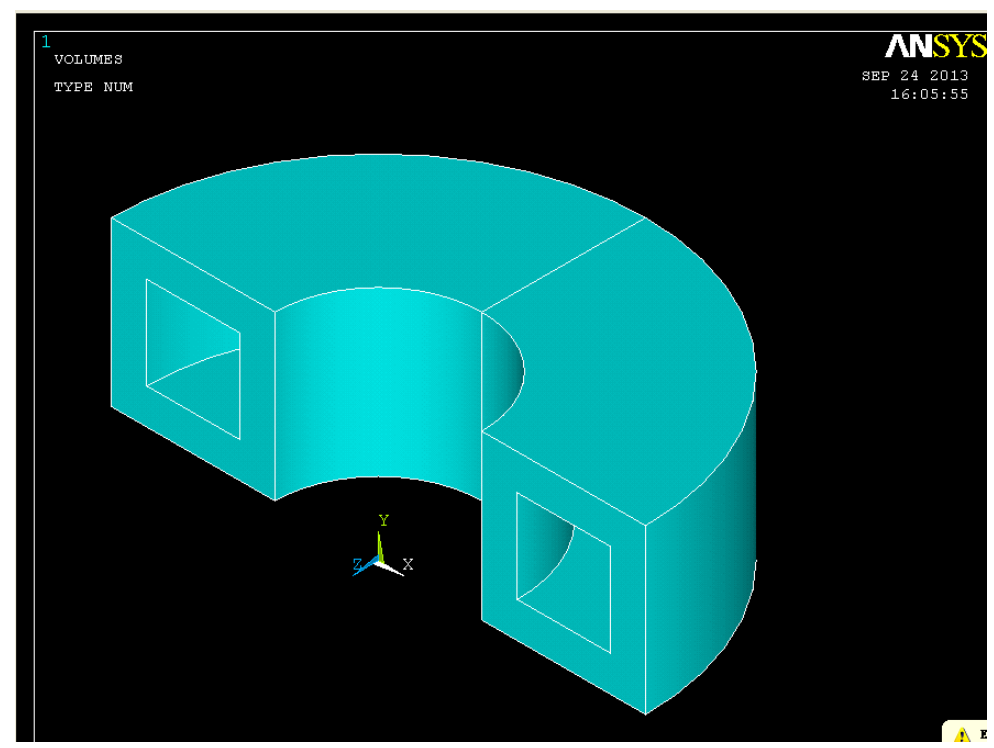

Figure 10. The model of magnetic shield.

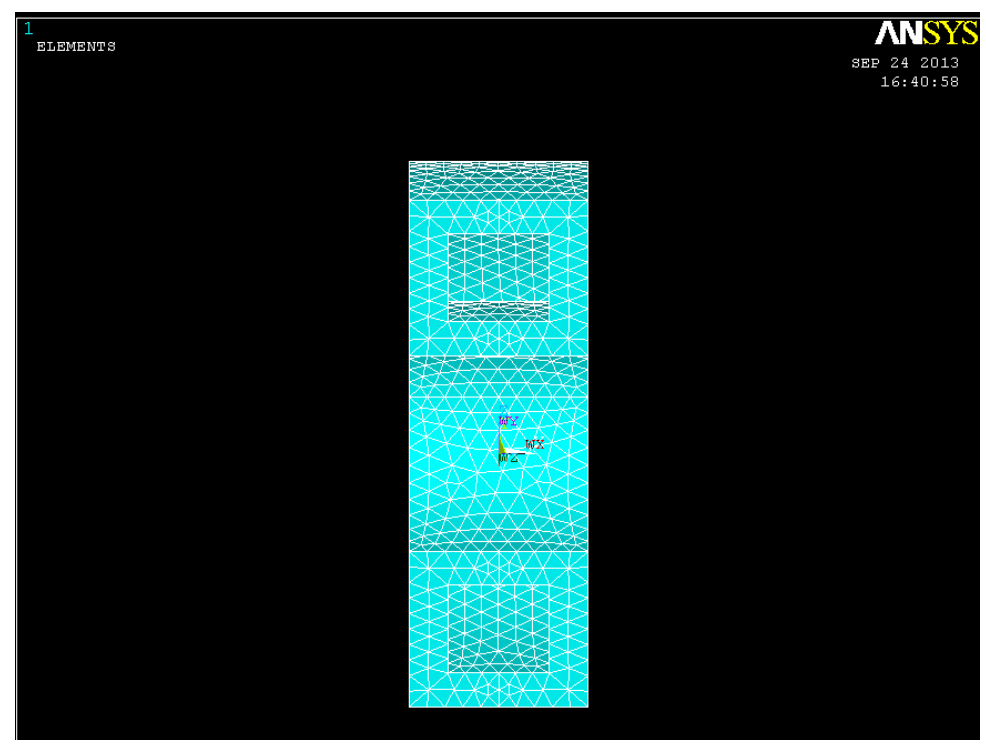

Figure 11. The subdivision of the shield in three-dimensional view. 


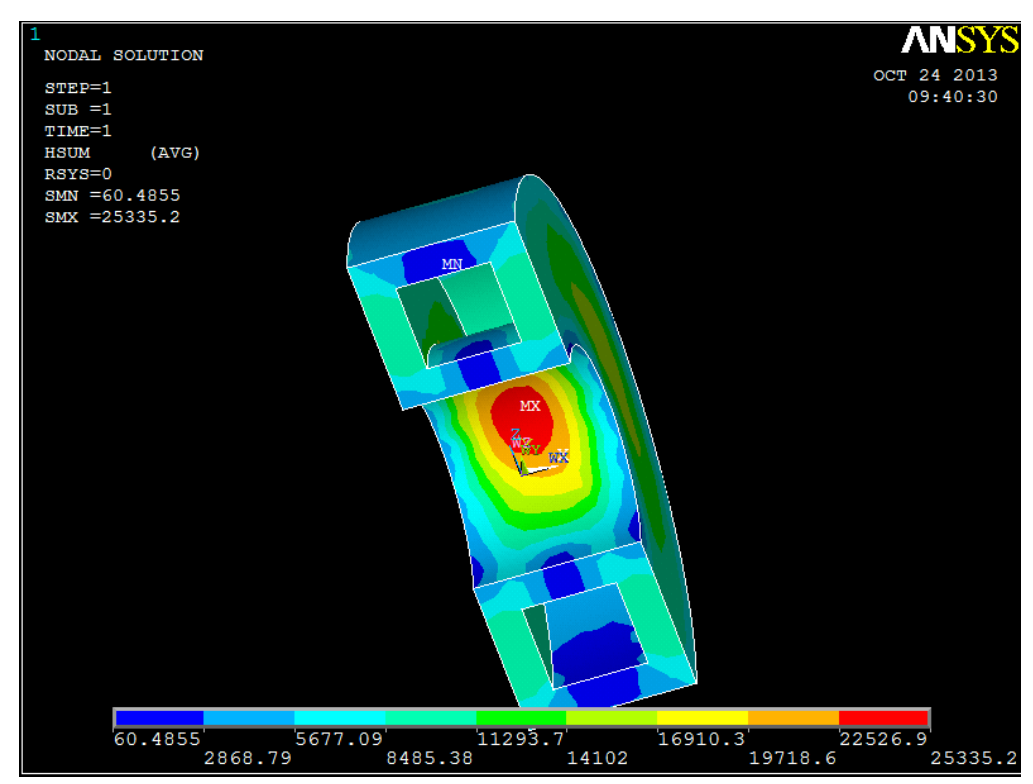

Figure 12. The distribution of the magnetic field in the external radial magnetic field.

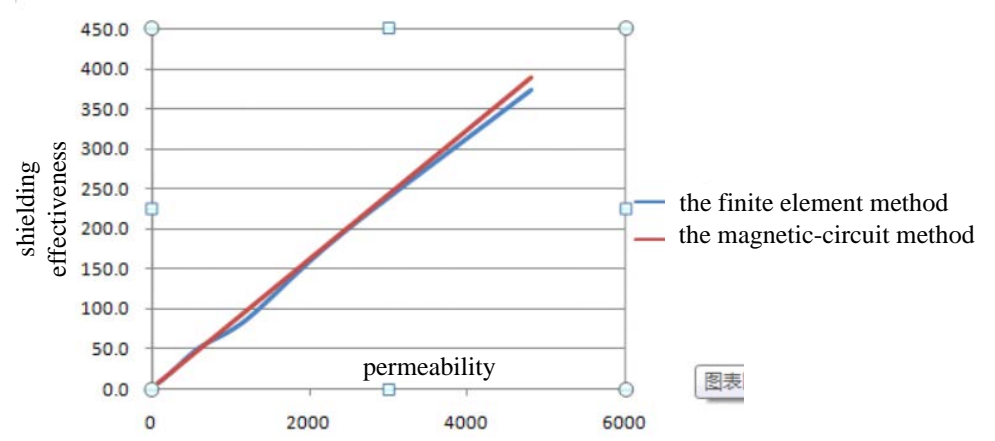

Figure 13. The relationship between the shielding effectiveness and permeability in the applied radial magnetic field.

The shielding effectiveness with different thickness of shield which is compared with the magnetic-circuit and the finite element method is shown in Figure 14.

As shown above, the shielding effectiveness will increase with the increase of the thickness of shield within the effective range. The results are similar by the two methods.

3) Different cross section length of shield

The shielding effectiveness with different cross section length of shield which is compared with the magnetic-circuit and the finite element method is shown in Figure 15.

As shown above, the shielding effectiveness with cross section length of shield is not the same with the two methods, however, both of the two curves are flat, the rate is almost zero when the length is over 6 . It means that the shielding effectiveness has little influence with the cross section of shield in the applied radial magnetic field.

4) Different inner diameter of shield

The shielding effectiveness with different inner diameter of shield which is compared with the magnetic-circuit and the finite element method is shown in Figure 16.

As shown above, the shielding effectiveness with inner diameter of shield in the applied radial magnetic field is similar by the two methods, but the curve with the finite element method is fluctuate, this should be attributed to the path to pick up when we use the software. 


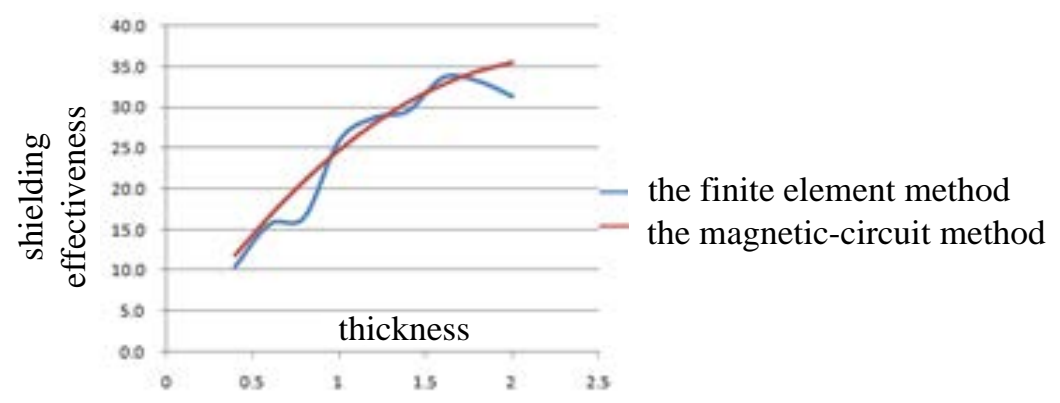

Figure 14. The relationship between the shielding effectiveness and the thickness of shield in the applied radial magnetic field.

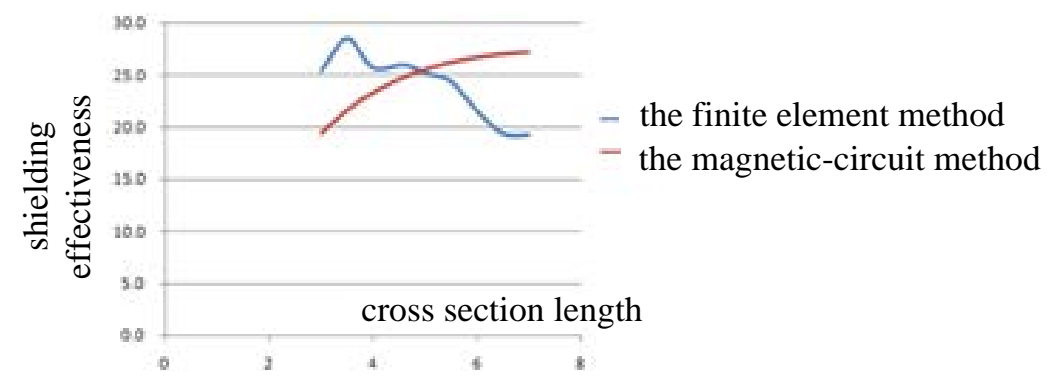

Figure 15. The relationship between the shielding effectiveness and the cross section length of shield in the applied radial magnetic field.

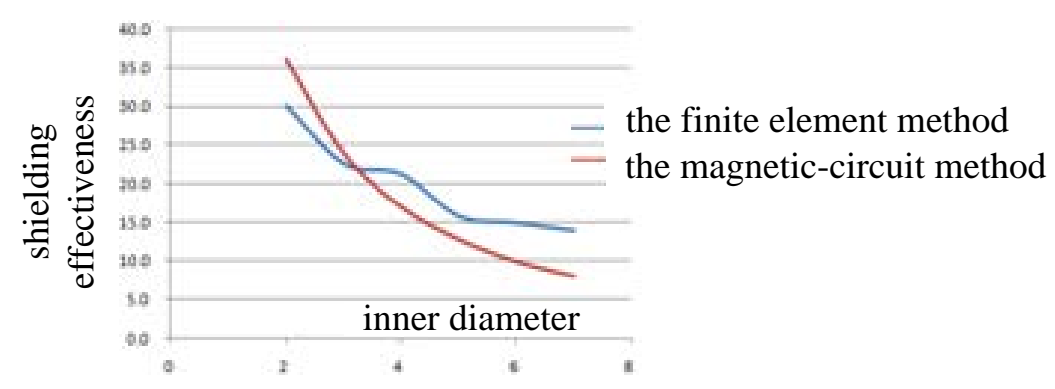

Figure 16. The relationship between the shielding effectiveness and the inner diameter of shield in the applied radial magnetic field.

\section{Conclusions}

The design of magnetic shielding is very important in the project of DCC, we haven't yet got effective result about magnetic shield so far. The influence factors of the shielding effectiveness are discussed in the paper, and also, the calculation and simulation results are compared with the actual situation, so there will be realistic instructive significance in the future.

Finally, the conclusion on the shielding efficiency can be obtained as follows.

1) The materials with high permeability should be used.

2) The thickness of shield should be controlled in a reasonable range.

3) The cross section length should be as small as possible within the effective range.

4) The inner diameter of shield should be designed to just hold the detecting coil.

5) It's effective to use the finite element method to analyze the shielding effectiveness.

\section{References}

[1] Ren, S., Ding, H., Li, M. and She, S. (1995) Magnetic Shielding Effectiveness for Comparators. IEEE Trans. Instrum. 
Meas.

[2] Shastry, S.V.K., Shamanna, K.N. and Katti, V.R. (1985) Shielding of Electromagnetic Fields of Current Sources by Hemispherical Enclosures. IEEE Trans. on Electromagnetic Compatibility.

[3] Kriezis, E.E. and Antonopoulos, C.S. (1984) Low-Frequency Electromagnetic Shielding in a System of Two Coaxial Cylindrical Shells. IEEE Trans. on Electromagnetic Compatibility, 26, 193-201.

[4] Moore, W.J.M. and Miljanic, P.N. (1988) The Current Comparator. Peter Peregrinus Ltd., London.

[5] Petersons, O. (1974) A Wide Range High Voltage Capacitance Bridge with One PPM Accuracy. D.Sc. Dissertation, School of Engineering and Applied Science, George Washington University, Washington, D.C.

[6] Wang, X.W. (n.d.) Study on Magnetic Shielding Effectiveness of Heavy Direct Current Sensor. Huazhong University of Science and Technology, Wuhan, 1-12. 